\title{
Statistical Distribution of Roots of a Polynomial Modulo Primes III
}

\author{
Yoshiyuki Kitaoka ${ }^{1}$ \\ Correspondence: Yoshiyuki Kitaoka, E-mail: kitaoka@meijo-u.ac.jp \\ Received: November 19, 2017 Accepted: December 5, 2017 Online Published: December 15, 2017 \\ doi:10.5539/ijsp.v7n1p115 \\ URL: https://doi.org/10.5539/ijsp.v7n1p115
}

\begin{abstract}
Let $f(x)=x^{n}+a_{n-1} x^{n-1}+\cdots+a_{0}\left(a_{n-1}, \ldots, a_{0} \in \mathbb{Z}\right)$ be a polynomial with complex roots $\alpha_{1}, \ldots, \alpha_{n}$ and suppose that a linear relation over $\mathbb{Q}$ among $1, \alpha_{1}, \ldots, \alpha_{n}$ is a multiple of $\sum_{i} \alpha_{i}+a_{n-1}=0$ only. For a prime number $p$ such that $f(x) \bmod p$ has $n$ distinct integer roots $0<r_{1}<\cdots<r_{n}<p$, we proposed in a previous paper a conjecture that the sequence of points $\left(r_{1} / p, \ldots, r_{n} / p\right)$ is equi-distributed in some sense. In this paper, we show that it implies the equi-distribution of the sequence of $r_{1} / p, \ldots, r_{n} / p$ in the ordinary sense and give the expected density of primes satisfying $r_{i} / p<a$ for a fixed suffix $i$ and $0<a<1$.
\end{abstract}

Keywords: polynomial, equi-distribution

\section{Introduction}

Let

$$
f(x)=x^{n}+a_{n-1} x^{n-1}+\cdots+a_{0}
$$

be a monic polynomial of degree $n(\geq 2)$ over the ring $\mathbb{Z}$ of integers with complex roots $\alpha_{1}, \ldots, \alpha_{n}$. We put

$$
\operatorname{Spl}_{X}(f):=\{p \leq X \mid f(x) \text { is fully splitting modulo } p\}
$$

for a positive number $X$ and $\operatorname{Spl}(f):=S p l_{\infty}(f)$. Here the letter $p$ denotes a prime number, and a polynomial $f(x)$ is fully splitting modulo $p$ if and only if

$$
f(x) \equiv \prod_{i=1}^{n}\left(x-r_{i}\right) \bmod p
$$

for some integers $r_{i}$. We know that $\operatorname{Spl}(f)$ is an infinite set and that the density theorem due to Chebotarev holds; that is,

$$
\lim _{X \rightarrow \infty} \frac{\# \operatorname{Spl}(f, X)}{\#\{p \leq X\}}=\frac{1}{[\mathbb{Q}(f): \mathbb{Q}]^{\prime}}
$$

where $\mathbb{Q}$ is the rational number field and $\mathbb{Q}(f)$ is a finite Galois extension field of $\mathbb{Q}$ generated by all roots of $f(x)$. In this note, we require the following condition on the above local roots $r_{1}, \ldots, r_{n}$ :

$$
0 \leq r_{1} \leq r_{2} \leq \cdots \leq r_{n}<p .
$$

The condition (3) determines the $i$ th local root $r_{i}$ uniquely. As a basic assumption, we assume that there is no non-trivial linear relation over $\mathbb{Q}$ among roots $\alpha_{1}, \ldots, \alpha_{n}$ and 1 except for a trivial relation $\sum \alpha_{i}+a_{n-1}=0$ in this paper. We know that any irreducible polynomial of prime degree, or a polynomial $f$ of degree $n$ with $[\mathbb{Q}(f): \mathbb{Q}]=n$ ! has no non-trivial linear relation among roots and 1 . An irreducible polynomial $f$ of degree 4 has a non-trivial linear relation among roots and 1 if and only if $f(x)$ is of the form $g(h(x))$ for quadratic polynomials $g, h$ (Kitaoka, 2017). When the degree is greater than 5 , there is no such a simple classification.

We consider the following two kinds of uniformity: Put

$$
\hat{\mathfrak{D}}_{n}:=\left\{\left(x_{1} \ldots, x_{n}\right) \in[0,1)^{n} \mid 0 \leq x_{1} \leq \cdots \leq x_{n}<1, \sum_{i=1}^{n} x_{i} \in \mathbb{Z}\right\}
$$

which is on the union of hyper-planes defined by $\sum x_{i}=k \in \mathbb{Z}$ in $\mathbb{R}^{n}$ and for a set $D \subset[0,1)^{n}$ with $D=\overline{D^{\circ}}$

$$
\operatorname{Pr}_{D}(f, X):=\frac{\#\left\{p \in S p l_{X}(f) \mid\left(r_{1} / p, \ldots, r_{n} / p\right) \in D\right\}}{\# S p l_{X}(f)}
$$


where local roots $r_{i}$ are supposed to satisfy properties (2), (3). We proposed (Kitaoka, 2017)

\section{Conjecture 1}

$$
\lim _{X \rightarrow \infty} \operatorname{Pr}_{D}(f, X)=\frac{\operatorname{vol}\left(D \cap \hat{\mathfrak{D}}_{n}\right)}{\operatorname{vol}\left(\hat{\mathfrak{D}}_{n}\right)} .
$$

Here, "vol" is the volume on the hyper-plane in $\mathbb{R}^{n}$. On the other hand, the classical concept of the uniformity is

\section{Conjecture 2}

$$
\lim _{X \rightarrow \infty} \frac{\sum_{p \in S p l_{X}(f)} \#\left\{i \mid r_{i} / p \leq a, 1 \leq i \leq n\right\}}{n \cdot \# S p l_{X}(f)}=a
$$

for a real number $a \in[0,1)$.

Due to (Duke, Friedlander \& Iwaniec, 1995) and (Tóth, 2000), Conjecture 2 is true for a quadratic polynomial, however nothing is known if $n>2$.

We stated in (Kitaoka, 2017) that Conjecture 2 follows from Conjecture 1 as far as we checked by the Monte Carlo method. We give the rigorous proof here, that is,

Theorem 1. Let $f(x)$ be a monic polynomial over $\mathbb{Z}$ of degree $n$. Under the assumption that there is no non-trivial linear relation over $\mathbb{Q}$ among roots of $f(x)$ and 1 , Conjecture 1 implies Conjecture 2.

To prove this, putting $D_{i, a}:=\left\{\left(x_{1}, \ldots, x_{n}\right) \in[0,1)^{n} \mid x_{i} \leq a\right\}$ for a given number $a \in[0,1)$, we have only to show

$$
\sum_{i=1}^{n} \frac{\operatorname{vol}\left(D_{i, a} \cap \hat{\mathfrak{D}}_{n}\right)}{n \cdot \operatorname{vol}\left(\hat{\mathfrak{D}}_{n}\right)}=a
$$

by (Kitaoka, 2017). To show it, we evaluate $\frac{\operatorname{vol}\left(D_{i, a} \cap \hat{\mathfrak{D}}_{n}\right)}{\operatorname{vol}\left(\hat{\mathfrak{D}}_{n}\right)}$ (Proposition 1$)$, which gives as a by-product the density of primes $p$ satisfying $r_{i} / p<a$ :

Theorem 2. Let $f(x)$ be a monic polynomial over $\mathbb{Z}$ of degree $n$. Under the assumption that there is no non-trivial linear relation over $\mathbb{Q}$ among roots of $f(x)$ and 1 . Then Conjecture 1 implies for $1 \leq i \leq n$

$$
\begin{aligned}
& \lim _{X \rightarrow \infty} \frac{\#\left\{p \in S p l_{X}(f) \mid r_{i} / p<a\right\}}{\# S p l_{X}(f)} \\
= & \frac{1}{(n-1) !} \sum_{\substack{0 \leq l \leq n \\
1 \leq l \leq n-1}} \sum_{k=i}^{n}(-1)^{h+k+n}\left(\begin{array}{l}
n \\
k
\end{array}\right) \sum_{m=1}^{n-1}\left(\begin{array}{c}
k \\
n-h-m+l
\end{array}\right)\left(\begin{array}{l}
n-k \\
m-l
\end{array}\right) M(l-h a)^{n-1},
\end{aligned}
$$

where the binomial coefficient $\left(\begin{array}{c}A \\ B\end{array}\right)$ is supposed to vanish unless $0 \leq B \leq A$, and $M(x):=\max (x, 0)$.

When $i=1$, a simpler formula is given in Proposition 1 in the next section. Let us give numerical data for a polynomial $f(x)=x^{6}+x^{5}+\cdots+1=\left(x^{7}-1\right) /(x-1)$. Put

$$
\operatorname{Ex}(a, m, i):=\frac{\#\left\{p \in \operatorname{Spl}_{X}(f) \mid r_{i} / p<a\right\}}{\# S p l_{X}(f)} \quad\left(X=10^{10} \cdot m\right)
$$

and denote the expected limit given by the above theorem by $T(a, i)$ and the error by

$$
\operatorname{er}(m):=10^{5} \max _{1 \leq k \leq 100,1 \leq i \leq 6}|\operatorname{Ex}(k / 100, m, i)-T(k / 100, i)| .
$$

The graph of $\operatorname{er}(m)(m=1, \ldots, 300)$ is below.

Conjecture 1 is generalized to a polynomial with a non-trivial linear relation among roots (Kitaoka, 2017). To treat such a polynomial, a more intrinsic proof of Theorem 1 independent of evaluation is desirable.

\section{Proof}

Hereafter, a real number $a$ satisfies $0 \leq a<1$. 


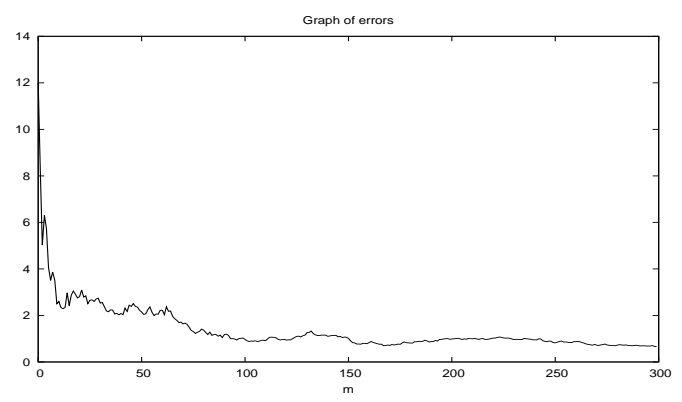

Lemma 1. For an integer $k$ with $1 \leq k \leq n$, let

$$
V(k):=\operatorname{vol}\left(\left\{x \in[0,1)^{n} \mid x_{1}, \ldots, x_{k} \leq a<x_{k+1}, \ldots, x_{n}, \sum_{j=1}^{n} x_{j} \in \mathbb{Z}\right\}\right) \cos \theta,
$$

for the angle $\theta$ of two hyper-planes defined by $x_{j}=0$ and by $x_{1}+\cdots+x_{n}=0$ in $\mathbb{R}^{n}$. Then we have

$$
\frac{\operatorname{vol}\left(D_{i, a} \cap \hat{\mathfrak{D}}_{n}\right)}{\operatorname{vol}\left(\hat{\mathfrak{D}}_{n}\right)}=\sum_{k=i}^{n}\left(\begin{array}{l}
n \\
k
\end{array}\right) V(k) \text {. }
$$

Proof. It is easy to see

$$
\begin{aligned}
& \operatorname{vol}\left(D_{i, a} \cap \hat{\mathfrak{D}}_{n}\right) \\
= & \sum_{k=i}^{n} \operatorname{vol}\left\{x \mid 0 \leq x_{1} \leq \cdots \leq x_{k} \leq a<x_{k+1} \leq \cdots \leq x_{n}<1, \sum x_{j} \in \mathbb{Z}\right\} \\
= & \sum_{k=i}^{n} \frac{1}{k !(n-k) !} \operatorname{vol}\left\{x \mid 0 \leq x_{1}, \ldots, x_{k} \leq a<x_{k+1}, \ldots, x_{n}<1, \sum x_{j} \in \mathbb{Z}\right\} \\
= & \frac{1}{n !} \sum_{k=i}^{n}\left(\begin{array}{l}
n \\
k
\end{array}\right) \operatorname{vol}\left\{x \mid 0 \leq x_{1}, \ldots, x_{k} \leq a<x_{k+1}, \ldots, x_{n}<1, \sum_{j} x_{j} \in \mathbb{Z}\right\} \\
= & \operatorname{vol}\left(\hat{\mathfrak{D}}_{n}\right) \sum_{k=i}^{n}\left(\begin{array}{l}
n \\
k
\end{array}\right) V(k),
\end{aligned}
$$

using $\operatorname{vol}\left(\hat{\mathfrak{D}}_{n}\right)=\frac{1}{n ! \cos \theta}$.

To evaluate $V(k)$, we quote the following (Feller, 1966):

Lemma 2. For a natural number $k$, the volume of a subset of the unit cube $[0,1)^{k}$ defined by $\left\{\left(x_{1}, \ldots, x_{k}\right) \mid x_{1}+\cdots+x_{k} \leq x\right\}$ is given by

$$
U_{k}(x):=\frac{1}{k !} \sum_{i=0}^{k}(-1)^{i}\left(\begin{array}{l}
k \\
i
\end{array}\right) M(x-i)^{k} .
$$

Lemma 3. For $k=n$, we have

$$
V(n)=\frac{1}{(n-1) !} \sum_{\substack{0 \leq i \leq n, n \\
1 \leq k \leq n-1}}(-1)^{i}\left(\begin{array}{c}
n \\
i
\end{array}\right) M(k-i a)^{n-1}
$$

Proof. It is easy to see that

$$
\begin{aligned}
V(n) & =\operatorname{vol}\left(\left\{x \in \mathbb{R}^{n} \mid 0 \leq x_{1}, \ldots, x_{n} \leq a, \sum_{i=1}^{n} x_{i} \in \mathbb{Z}\right\}\right) \cos \theta \\
& =\sum_{k=1}^{n-1} \operatorname{vol}\left(\left\{x \in \mathbb{R}^{n} \mid 0 \leq x_{1}, \ldots, x_{n} \leq a, \sum_{i=1}^{n} x_{i}=k\right\}\right) \cos \theta
\end{aligned}
$$




$$
\begin{aligned}
& =\sum_{k=1}^{n-1} \operatorname{vol}\left(\left\{x \in \mathbb{R}^{n-1} \mid 0 \leq x_{1}, \ldots, x_{n-1} \leq a, 0 \leq k-\sum_{i=1}^{n-1} x_{i} \leq a\right\}\right) \\
& =\sum_{k=1}^{n-1} \operatorname{vol}\left(\left\{x \in \mathbb{R}^{n-1} \mid 0 \leq x_{1}, \ldots, x_{n-1} \leq a, \sum_{i=1}^{n-1} x_{i} \leq k\right\}\right) \\
& \quad-\sum_{k=1}^{n-1} \operatorname{vol}\left(\left\{x \in \mathbb{R}^{n-1} \mid 0 \leq x_{1}, \ldots, x_{n-1} \leq a, \sum_{i=1}^{n-1} x_{i} \leq k-a\right\}\right) .
\end{aligned}
$$

The volume of the set $\left\{x \in \mathbb{R}^{n-1} \mid 0 \leq x_{1}, \ldots, x_{n-1} \leq a, \sum_{i=1}^{n-1} x_{i} \leq K\right\}$ is equal to

$$
\begin{aligned}
& a^{n-1} \operatorname{vol}\left(\left\{x \in \mathbb{R}^{n-1} \mid 0 \leq t_{1}, \ldots, t_{n-1} \leq 1, \sum_{i=1}^{n-1} t_{i} \leq K / a\right\}\right) \\
& =a^{n-1} U_{n-1}(K / a) \\
& =\frac{a^{n-1}}{(n-1) !} \sum_{i=0}^{n-1}(-1)^{i}\left(\begin{array}{c}
n-1 \\
i
\end{array}\right) M(K / a-i)^{n-1} \\
& =\frac{1}{(n-1) !} \sum_{i=0}^{n-1}(-1)^{i}\left(\begin{array}{c}
n-1 \\
i
\end{array}\right) M(K-i a)^{n-1} \text {. }
\end{aligned}
$$

Therefore we have

$$
\begin{aligned}
V(n) & =\frac{1}{(n-1) !} \sum_{k=1}^{n-1} \sum_{i=0}^{n-1}(-1)^{i}\left(\begin{array}{c}
n-1 \\
i
\end{array}\right)\left\{M(k-i a)^{n-1}-M(k-(i+1) a)^{n-1}\right\} \\
& =\frac{1}{(n-1) !} \sum_{k=1}^{n-1} \sum_{i=0}^{n-1}(-1)^{i}\left(\begin{array}{c}
n-1 \\
i
\end{array}\right) M(k-i a)^{n-1} \\
& +\frac{1}{(n-1) !} \sum_{k=1}^{n-1} \sum_{i=1}^{n}(-1)^{i}\left(\begin{array}{c}
n-1 \\
i-1
\end{array}\right) M(k-i a)^{n-1} \\
& =\frac{1}{(n-1) !} \sum_{k=1}^{n-1} \sum_{i=0}^{n}(-1)^{i}\left(\begin{array}{c}
n \\
i
\end{array}\right) M(k-i a)^{n-1} .
\end{aligned}
$$

Lemma 4. In case of $1 \leq k \leq n-1$, we have

$$
V(k)=\sum_{m=1}^{n-1}\left(U_{k, n-1}(m-a)-U_{k, n-1}(m-1)\right),
$$

where

$$
U_{k, r}(t):=\operatorname{vol}\left\{x \in[0,1)^{r} \mid x_{1}, \ldots, x_{k} \leq a<x_{k+1}, \ldots, x_{r}, \sum_{j=1}^{r} x_{j}<t\right\} .
$$

Proof. We see that

$$
\begin{aligned}
V(k) & =\sum_{m=1}^{n-1} v o l\left\{x \in[0,1)^{n} \mid x_{1}, \ldots, x_{k} \leq a<x_{k+1}, \ldots, x_{n}, \sum_{j=1}^{n} x_{j}=m\right\} \cos \theta \\
& =\sum_{m=1}^{n-1} v o l\left\{x \in[0,1)^{n-1} \mid x_{1}, \ldots, x_{k} \leq a<x_{k+1}, \ldots, x_{n-1}, a<m-\sum_{j=1}^{n-1} x_{j}<1\right\} \\
& =\sum_{m=1}^{n-1}\left(U_{k, n-1}(m-a)-U_{k, n-1}(m-1)\right) .
\end{aligned}
$$


Lemma 5. For integers $r, k$ with $1 \leq k \leq r$, we have

$$
U_{k, r+1}(t)=\int_{a}^{1} U_{k, r}(t-w) d w .
$$

Proof. This follows from the equation

$$
U_{k, r+1}(t)=\int_{x_{r+1}=a}^{1}\left(\int_{D} d x_{1}, \ldots d x_{r}\right) d x_{r+1}
$$

where the domain $D$ is given by the conditions $0 \leq x_{1}, \ldots, x_{k} \leq a<x_{k+1}, \ldots, x_{r}, \sum_{j=1}^{r} x_{j}<t-x_{r+1}$.

Lemma 6.

$$
\int_{a}^{1} M(t-w)^{m} d w=\frac{1}{m+1}\left\{M(t-a)^{m+1}-M(t-1)^{m+1}\right\}
$$

Proof. The left-hand side is equal to

$$
\begin{aligned}
& \int_{a}^{1} \max (t-w, 0)^{m} d w \\
= & \int_{t-a}^{t-1} \max (W, 0)^{m}(-d W) \\
= & -\int_{-\infty}^{t-1} \max (W, 0)^{m} d W+\int_{-\infty}^{t-a} \max (W, 0)^{m} d W \\
= & -\frac{1}{m+1} M(t-1)^{m+1}+\frac{1}{m+1} M(t-a)^{m+1} .
\end{aligned}
$$

Lemma 7. For integers $j, k$ with $j \geq 0, k \geq 1, U_{k, k+j}(t)$ is equal to

$$
\frac{1}{(k+j) !} \sum_{i=0}^{k}(-1)^{i}\left(\begin{array}{l}
k \\
i
\end{array}\right) \sum_{h=0}^{j}(-1)^{j+h}\left(\begin{array}{l}
j \\
h
\end{array}\right) M(t+h-j-(i+h) a)^{k+j} .
$$

Proof. Suppose that $j=0$; then $U_{k, k}(t)$ equals

$$
\begin{aligned}
& \operatorname{vol}\left\{x \in[0,1)^{k} \mid x_{1}, \ldots, x_{k} \leq a, \sum_{j=1}^{k} x_{j}<t\right\} \\
= & a^{k} U_{k}(t / a) \\
= & \frac{1}{k !} \sum_{i=0}^{k}(-1)^{i}\left(\begin{array}{l}
k \\
i
\end{array}\right) M(t-i a)^{k} .
\end{aligned}
$$

Second, suppose that the equation (13) is true; then we see that $U_{k, k+j+1}(t)$ equals

$$
\begin{aligned}
& \int_{a}^{1} U_{k, k+j}(t-w) d w \\
= & \frac{1}{(k+j) !} \sum_{i=0}^{k}(-1)^{i}\left(\begin{array}{l}
k \\
i
\end{array}\right) \sum_{h=0}^{j}(-1)^{j+h}\left(\begin{array}{l}
j \\
h
\end{array}\right) \int_{a}^{1} M(t-w+h-j-(i+h) a)^{k+j} d w \\
= & \frac{1}{(k+j) !} \sum_{i=0}^{k}(-1)^{i}\left(\begin{array}{l}
k \\
i
\end{array}\right) \sum_{h=0}^{j}(-1)^{j+h}\left(\begin{array}{l}
j \\
h
\end{array}\right) \times
\end{aligned}
$$




$$
\begin{aligned}
& \frac{1}{k+j+1}\left\{M(t+h-j-(i+h+1) a)^{k+j+1}-M(t+h-j-1-(i+h) a)^{k+j+1}\right\} \\
= & \frac{1}{(k+j+1) !} \sum_{i=0}^{k}(-1)^{i}\left(\begin{array}{l}
k \\
i
\end{array}\right) \times \\
& \left\{\sum_{h=1}^{j+1}(-1)^{j+h+1}\left(\begin{array}{c}
j \\
h-1
\end{array}\right) M(t+h-j-1-(i+h) a)^{k+j+1}-\sum_{h=0}^{j}(-1)^{j+h}\left(\begin{array}{l}
j \\
h
\end{array}\right) M(t+h-j-1-(i+h) a)^{k+j+1}\right\} \\
= & \frac{1}{(k+j+1) !} \sum_{i=0}^{k}(-1)^{i}\left(\begin{array}{l}
k \\
i
\end{array}\right) \times\left\{\begin{array}{c}
j \\
\sum_{h=1}^{j}(-1)^{j+h+1}\left(\left(\begin{array}{c}
j \\
h-1
\end{array}\right)+\left(\begin{array}{l}
j \\
h
\end{array}\right)\right) M(t+h-j-1-(i+h) a)^{k+j+1}
\end{array} \quad+M(t-(i+j+1) a)^{k+j+1}-(-1)^{j} M(t-j-1-i a)^{k+j+1}\right\} \\
= & \frac{1}{(k+j+1) !} \sum_{i=0}^{k}\left(\begin{array}{c}
(-1)^{i} \\
\left(\begin{array}{l}
k \\
i
\end{array}\right) \times \quad \begin{array}{c}
j \\
\end{array}
\end{array}\right. \\
& \left\{\begin{array}{c}
j \\
\left.\sum_{h=1}(-1)^{j+h+1}\left(\begin{array}{c}
j+1 \\
h
\end{array}\right) M(t+h-j-1-(i+h) a)^{k+j+1}+M(t-(i+j+1) a)^{k+j+1}-(-1)^{j} M(t-j-1-i a)^{k+j+1}\right\},
\end{array}\right.
\end{aligned}
$$

which completes the induction.

Lemma 8. For $1 \leq k \leq n-1$, we have

$$
V(k)=\frac{1}{(n-1) !} \sum_{\substack{0 \leq n \leq n \\
1 \leq l \leq n-1}}(-1)^{n+k+h} \sum_{m=1}^{n-1}\left(\begin{array}{c}
k \\
n-h-m+l
\end{array}\right)\left(\begin{array}{c}
n-k \\
m-l
\end{array}\right) M(l-h a)^{n-1} .
$$

Proof. For $1 \leq k, m \leq n-1$, we have

$$
\begin{aligned}
& (n-1) !\left\{U_{k, n-1}(m-a)-U_{k, n-1}(m-1)\right\} \\
= & \sum_{i, h \in \mathbb{Z}}(-1)^{i}\left(\begin{array}{l}
k \\
i
\end{array}\right)(-1)^{n-1-k+h}\left(\begin{array}{c}
n-1-k \\
h
\end{array}\right) M(m-a+h-(n-1-k)-(i+h) a)^{n-1} \\
& \quad-\sum_{i, h \in \mathbb{Z}}(-1)^{i}\left(\begin{array}{c}
k \\
i
\end{array}\right)(-1)^{n-1-k+h}\left(\begin{array}{c}
n-1-k \\
h
\end{array}\right) M(m-1+h-(n-1-k)-(i+h) a)^{n-1} \\
= & \sum_{h, l \in \mathbb{Z}}(-1)^{n+k+h}\left(\begin{array}{c}
k \\
n+l-h-m
\end{array}\right)\left\{\left(\begin{array}{c}
n-1-k \\
m-l
\end{array}\right)+\left(\begin{array}{c}
n-1-k \\
m-l-1
\end{array}\right)\right\} M(l-h a)^{n-1} \\
= & \sum_{\substack{0 \leq h \leq n, 1 \\
1 \leq l \leq n-1}}(-1)^{n+k+h}\left(\begin{array}{c}
k \\
n+l-h-m
\end{array}\right)\left(\begin{array}{c}
n-k \\
m-l
\end{array}\right) M(l-h a)^{n-1},
\end{aligned}
$$

where the restrictions on $h, l$ follow from conditions $1 \leq k, m \leq n-1,0 \leq n+l-h-m \leq k, 0 \leq m-l \leq n-k$. Lemma 4 completes the proof.

Lemma 9. Let $m, n$ be integers satisfying $0 \leq m \leq n-1$. Then we have

$$
\sum_{k=0}^{m}(-1)^{k}\left(\begin{array}{l}
n \\
k
\end{array}\right)=(-1)^{m}\left(\begin{array}{c}
n-1 \\
m
\end{array}\right)
$$

For a polynomial $P(x)=c_{n} x^{n}+\cdots+c_{0}$, we have

$$
\sum_{k=0}^{n}(-1)^{k} P(k)\left(\begin{array}{l}
n \\
k
\end{array}\right)=c_{n}(-1)^{n} n !
$$

These are well-known and we omit the proof. 
Proposition 1. For an integer $i$ with $1 \leq i \leq n$ and a real number $a \in[0,1)$, we have

$$
\begin{aligned}
& (n-1) ! \operatorname{vol}\left(D_{i, a} \cap \hat{\mathfrak{D}}_{n}\right) / \operatorname{vol}\left(\hat{\mathfrak{D}}_{n}\right) \\
= & \sum_{\substack{0 \leq l \leq n \\
1 \leq l \leq n-1}} \sum_{k=i}^{n}(-1)^{h+k+n}\left(\begin{array}{l}
n \\
k
\end{array}\right)\left(\left(\begin{array}{l}
n \\
h
\end{array}\right)-\sum_{h \leq q \leq \max (l, h-1)}\left(\begin{array}{c}
k \\
q-h
\end{array}\right)\left(\begin{array}{l}
n-k \\
n-q
\end{array}\right)\right) M(l-h a)^{n-1} .
\end{aligned}
$$

In particular, we have for $i=1$

$$
\operatorname{vol}\left(D_{1, a} \cap \hat{\mathfrak{D}}_{n}\right) / \operatorname{vol}\left(\hat{\mathfrak{D}}_{n}\right)=\sum_{\substack{0 \leq h \leq n, 1 \leq l \leq n-1}} C_{1}(l, h) M(l-h a)^{n-1},
$$

where

$$
C_{1}(l, h)=\frac{1}{(n-1) !} \begin{cases}(-1)^{n+h+1}\left(\begin{array}{c}
n \\
h
\end{array}\right) & \text { if } h \geq l+1, \\
0 & \text { if } 1 \leq h \leq l, \\
(-1)^{n+l+1}\left(\begin{array}{c}
n-1 \\
l
\end{array}\right) & \text { if } h=0 .\end{cases}
$$

Proof. By Lemma 1, we have

$$
\begin{aligned}
& (n-1) ! \operatorname{vol}\left(D_{i, a} \cap \hat{\mathfrak{D}}_{n}\right) / \operatorname{vol}\left(\hat{\mathfrak{D}}_{n}\right) \\
= & (n-1) ! \sum_{k=i}^{n}\left(\begin{array}{l}
n \\
k
\end{array}\right) V(k) \\
= & (n-1) ! V(n)+(n-1) ! \sum_{k=i}^{n-1}\left(\begin{array}{l}
n \\
k
\end{array}\right) V(k) \\
= & \sum_{\substack{0 \leq l \leq n \\
1 \leq l \leq n-1}}(-1)^{h}\left(\begin{array}{l}
n \\
h
\end{array}\right) M(l-h a)^{n-1} \\
+ & \sum_{k=i}^{n-1}\left(\begin{array}{l}
n \\
k
\end{array}\right) \sum_{\substack{0 \leq \leq \leq n \\
1 \leq l \leq n-1}}(-1)^{n+k+h} \sum_{m=1}^{n-1}\left(\begin{array}{c}
k \\
n-h-m+l
\end{array}\right)\left(\begin{array}{l}
n-k \\
m-l
\end{array}\right) M(l-h a)^{n-1} \\
= & \sum_{k=i}^{n}\left(\begin{array}{l}
n \\
k
\end{array}\right) \sum_{\substack{0 \leq \leq \leq n \\
1 \leq l \leq n-1}}(-1)^{n+k+h} \sum_{m=1}^{n-1}\left(\begin{array}{c}
k \\
n-h-m+l
\end{array}\right)\left(\begin{array}{l}
n-k \\
m-l
\end{array}\right) M(l-h a)^{n-1},
\end{aligned}
$$

since the binomial coefficient $\left(\begin{array}{c}0 \\ m-l\end{array}\right)$ vanishes unless $m=l$. The partial sum $\sum_{m=1}^{n-1}\left(\begin{array}{c}k \\ n-h-m+l\end{array}\right)\left(\begin{array}{l}n-k \\ m-l\end{array}\right)$ is equal to

$$
\begin{aligned}
& \sum_{1-l \leq q \leq n-1-l}\left(\begin{array}{c}
k \\
n-h-q
\end{array}\right)\left(\begin{array}{c}
n-k \\
q
\end{array}\right) \\
= & \sum_{0 \leq q \leq \min (n-1-l, n-h)}\left(\begin{array}{c}
k \\
n-h-q
\end{array}\right)\left(\begin{array}{c}
n-k \\
q
\end{array}\right) \\
= & \left(\begin{array}{c}
n \\
n-h
\end{array}\right)-\sum_{\min (n-1-l, n-h)+1 \leq q \leq n-h}\left(\begin{array}{c}
k \\
n-h-q
\end{array}\right)\left(\begin{array}{c}
n-k \\
q
\end{array}\right) \\
= & \left(\begin{array}{l}
n \\
h
\end{array}\right)-\sum_{h \leq q \leq \max (l, h-1)}\left(\begin{array}{c}
k \\
q-h
\end{array}\right)\left(\begin{array}{l}
n-k \\
n-q
\end{array}\right) .
\end{aligned}
$$

Let us assume that $i=1$ to show (16). Putting

$$
\begin{aligned}
T(l, h) & :=\sum_{k=1}^{n}(-1)^{h+k+n}\left(\begin{array}{l}
n \\
k
\end{array}\right)\left(\left(\begin{array}{l}
n \\
h
\end{array}\right)-\sum_{h \leq q \leq \max (l, h-1)}\left(\begin{array}{c}
k \\
q-h
\end{array}\right)\left(\begin{array}{l}
n-k \\
n-q
\end{array}\right)\right) \\
& =-(-1)^{h+n}\left(\begin{array}{l}
n \\
h
\end{array}\right)-\sum_{k=1}^{n}(-1)^{h+k+n}\left(\begin{array}{l}
n \\
k
\end{array}\right) \sum_{q=h}^{\max (l, h-1)}\left(\begin{array}{c}
k \\
q-h
\end{array}\right)\left(\begin{array}{l}
n-k \\
n-q
\end{array}\right),
\end{aligned}
$$


we have only to prove $T(l, h)=(n-1) ! C_{1}(l, h)$. It is obviously true if $h \geq l+1$, since the partial sum on $q$ is empty. In case of $h=0$, we see that $T(l, 0)$ is equal to

$$
\begin{aligned}
& -(-1)^{n}-\sum_{k=1}^{n}(-1)^{k+n}\left(\begin{array}{l}
n \\
k
\end{array}\right) \sum_{q=0}^{l}\left(\begin{array}{l}
k \\
q
\end{array}\right)\left(\begin{array}{l}
n-k \\
n-q
\end{array}\right) \\
= & -(-1)^{n}-\sum_{k=1}^{n}(-1)^{k+n}\left(\begin{array}{l}
n \\
k
\end{array}\right) \sum_{q=0}^{l} \delta_{k, q} \\
= & -(-1)^{n}-\sum_{k=1}^{l}(-1)^{k+n}\left(\begin{array}{l}
n \\
k
\end{array}\right) \\
= & -\sum_{k=0}^{l}(-1)^{k+n}\left(\begin{array}{l}
n \\
k
\end{array}\right) \\
= & -(-1)^{n+1}\left(\begin{array}{c}
n-1 \\
l
\end{array}\right) .
\end{aligned}
$$

Lastly assume that $1 \leq h \leq l$. The sum $T(l, h)+(-1)^{h+n}\left(\begin{array}{c}n \\ h\end{array}\right)$ is equal to

$$
\begin{aligned}
& -\sum_{k=1}^{n}(-1)^{h+k+n}\left(\begin{array}{l}
n \\
k
\end{array}\right) \sum_{q=h}^{l}\left(\begin{array}{c}
k \\
q-h
\end{array}\right)\left(\begin{array}{l}
n-k \\
n-q
\end{array}\right) \\
= & -\sum_{q=h}^{l}(-1)^{h+n}\left(\begin{array}{l}
n \\
h
\end{array}\right)\left(\begin{array}{l}
n-h \\
q-h
\end{array}\right) \sum_{k=1}^{n}(-1)^{k}\left(\begin{array}{c}
h \\
q-k
\end{array}\right) \\
= & -\sum_{q=h}^{l}(-1)^{h+n}\left(\begin{array}{l}
n \\
h
\end{array}\right)\left(\begin{array}{l}
n-h \\
q-h
\end{array}\right)(-1)^{q} \sum_{K=0}^{q-1}(-1)^{K}\left(\begin{array}{l}
h \\
K
\end{array}\right) \\
= & -\sum_{q=h}^{l}(-1)^{h+n}\left(\begin{array}{l}
n \\
h
\end{array}\right)\left(\begin{array}{l}
n-h \\
q-h
\end{array}\right)(-1)^{q}(-1)^{q-1}\left(\begin{array}{l}
h-1 \\
q-1
\end{array}\right) \\
= & \sum_{q=h}^{l}(-1)^{h+n}\left(\begin{array}{l}
n \\
h
\end{array}\right)\left(\begin{array}{l}
n-h \\
q-h
\end{array}\right)\left(\begin{array}{l}
h-1 \\
q-1
\end{array}\right) \\
= & (-1)^{h+n}\left(\begin{array}{l}
n \\
h
\end{array}\right)^{l}
\end{aligned}
$$

which implies $T(l, h)=0$.

The proposition gives Theorem 2 by (17), and we see that the left-hand side of (7) is the sum of $C(l, h) M(l-h a)^{n-1}$ over integers $l, h$ satisfying

$$
1 \leq l \leq n-1,0 \leq h \leq n,
$$

where

$$
\begin{aligned}
C(l, h) & :=\frac{1}{n !} \sum_{1 \leq i \leq k \leq n}(-1)^{h+k+n}\left(\begin{array}{l}
n \\
k
\end{array}\right)\left(\left(\begin{array}{l}
n \\
h
\end{array}\right)-\sum_{h \leq q \leq \max (l, h-1)}\left(\begin{array}{c}
k \\
q-h
\end{array}\right)\left(\begin{array}{l}
n-k \\
n-q
\end{array}\right)\right) \\
& =\frac{1}{n !} \sum_{0 \leq k \leq n}(-1)^{h+k+n} k\left(\begin{array}{l}
n \\
k
\end{array}\right)\left(\left(\begin{array}{l}
n \\
h
\end{array}\right)-\sum_{h \leq q \leq \max (l, h-1)}\left(\begin{array}{c}
k \\
q-h
\end{array}\right)\left(\begin{array}{l}
n-k \\
n-q
\end{array}\right)\right) \\
& =\frac{-1}{n !} \sum_{0 \leq k \leq n}(-1)^{h+k+n} k\left(\begin{array}{l}
n \\
k
\end{array}\right) \sum_{h \leq q \leq \max (l, h-1)}\left(\begin{array}{c}
k \\
q-h
\end{array}\right)\left(\begin{array}{l}
n-k \\
n-q
\end{array}\right) .
\end{aligned}
$$


To prove (7), we will show

$$
C(l, h)= \begin{cases}\frac{-(-1)^{n-l}}{(n-1) !}\left(\begin{array}{c}
n-2 \\
l-1
\end{array}\right) & \text { if } h=0, \\
\frac{(-1)^{n-l}}{(n-1) !}\left(\begin{array}{c}
n-2 \\
l-1
\end{array}\right) & \text { if } h=1, \\
0 & \text { if } h \geq 2 .\end{cases}
$$

Under the equations (20), Theorem 1 is proved as follows: The left-hand side of (7) is equal to

$$
\begin{aligned}
& \sum_{l=1}^{n-1} \frac{-(-1)^{n-l}}{(n-1) !}\left(\begin{array}{c}
n-2 \\
l-1
\end{array}\right) M(l)^{n-1}+\sum_{l=1}^{n-1} \frac{(-1)^{n-l}}{(n-1) !}\left(\begin{array}{c}
n-2 \\
l-1
\end{array}\right) M(l-a)^{n-1} \\
= & \sum_{l=1}^{n-1} \frac{-(-1)^{n-l}}{(n-1) !}\left(\begin{array}{c}
n-2 \\
l-1
\end{array}\right)\left(l^{n-1}-(l-a)^{n-1}\right) \\
= & \sum_{l=0}^{n-2} \frac{(-1)^{n+l}}{(n-1) !}\left(\begin{array}{c}
n-2 \\
l
\end{array}\right)\left((n-1) a l^{n-2}+O\left(l^{n-3}\right)\right) \\
= & a .
\end{aligned}
$$

Suppose $h=0$; we see that

$$
\begin{aligned}
C(l, 0) & =\frac{-1}{n !} \sum_{0 \leq k \leq n}(-1)^{k+n} k\left(\begin{array}{l}
n \\
k
\end{array}\right) \sum_{0 \leq q \leq l}\left(\begin{array}{l}
k \\
q
\end{array}\right)\left(\begin{array}{l}
n-k \\
n-q
\end{array}\right) \\
& =\frac{-1}{n !} \sum_{0 \leq k \leq n}(-1)^{k+n} k\left(\begin{array}{l}
n \\
k
\end{array}\right) \sum_{0 \leq q \leq l} \delta_{k, q} \\
& =\frac{-1}{n !} \sum_{0 \leq k \leq l}(-1)^{k+n} k\left(\begin{array}{l}
n \\
k
\end{array}\right) \\
& =\frac{-1}{(n-1) !} \sum_{0 \leq k \leq l}(-1)^{k+n}\left(\begin{array}{c}
n-1 \\
k-1
\end{array}\right) \\
& =\frac{-1}{(n-1) !} \sum_{0 \leq k \leq l-1}(-1)^{k+n+1}\left(\begin{array}{c}
n-1 \\
k
\end{array}\right) \\
& =\frac{(-1)^{n+l+1}}{(n-1) !}\left(\begin{array}{c}
n-2 \\
l-1
\end{array}\right),
\end{aligned}
$$

which is (20).

Second we see that

$$
C(l, 1)=\frac{-1}{n !} \sum_{0 \leq k \leq n}(-1)^{1+k+n} k\left(\begin{array}{l}
n \\
k
\end{array}\right) \sum_{1 \leq q \leq l}\left(\begin{array}{c}
k \\
q-1
\end{array}\right)\left(\begin{array}{l}
n-k \\
n-q
\end{array}\right) .
$$

Unless $q-1 \leq k$ and $n-q \leq n-k$, binomial coefficients vanish, hence we may assume that $q=k$ or $q=k+1$, and we see

$$
\begin{aligned}
C(l, 1) & =\frac{-1}{n !} \sum_{0 \leq k \leq n}(-1)^{1+k+n} k\left(\begin{array}{l}
n \\
k
\end{array}\right) \sum_{1 \leq q \leq l}\left(k \delta_{q, k}+(n-k) \delta_{q, k+1}\right) \\
& =\frac{-1}{n !} \sum_{0 \leq k \leq l}(-1)^{1+k+n} k^{2}\left(\begin{array}{l}
n \\
k
\end{array}\right)+\frac{-1}{n !} \sum_{0 \leq k \leq l-1}(-1)^{1+k+n} k(n-k)\left(\begin{array}{l}
n \\
k
\end{array}\right) \\
& =\frac{(-1)^{n}}{(n-1) !} \sum_{0 \leq k \leq l-1}(-1)^{k} k\left(\begin{array}{l}
n \\
k
\end{array}\right)+\frac{1}{n !}(-1)^{l+n} 2^{2}\left(\begin{array}{l}
n \\
l
\end{array}\right)
\end{aligned}
$$




$$
\begin{aligned}
& =\frac{(-1)^{n} n}{(n-1) !} \sum_{0 \leq k \leq l-1}(-1)^{k}\left(\begin{array}{l}
n-1 \\
k-1
\end{array}\right)+\frac{1}{n !}(-1)^{l+n} l^{2}\left(\begin{array}{l}
n \\
l
\end{array}\right) \\
& =\frac{(-1)^{n+1} n}{(n-1) !}(-1)^{l-2}\left(\begin{array}{c}
n-2 \\
l-2
\end{array}\right)+\frac{1}{n !}(-1)^{l+n} l^{2}\left(\begin{array}{l}
n \\
l
\end{array}\right) \\
& =\frac{(-1)^{n+l}}{(n-1) !}\left(\begin{array}{l}
n-2 \\
l-1
\end{array}\right) .
\end{aligned}
$$

Finally, assume that $h \geq 2$; hence $1 \leq l \leq n-1,2 \leq h \leq n$ are supposed. By (19), we have

$$
\begin{aligned}
& -n ! C(l, h) \\
= & \sum_{h \leq q \leq \max (l, h-1)}(-1)^{h+n}\left(\begin{array}{l}
n \\
h
\end{array}\right)\left(\begin{array}{l}
n-h \\
n-q
\end{array}\right) \sum_{0 \leq k \leq n}(-1)^{k} k\left(\begin{array}{c}
h \\
q-k
\end{array}\right) \\
= & \sum_{h \leq q \leq \max (l, h-1)}(-1)^{h+n}\left(\begin{array}{l}
n \\
h
\end{array}\right)\left(\begin{array}{l}
n-h \\
n-q
\end{array}\right) \sum_{0 \leq K \leq q}(-1)^{q+K}(q-K)\left(\begin{array}{l}
h \\
K
\end{array}\right) \\
= & 0,
\end{aligned}
$$

since

$$
\sum_{0 \leq K \leq q}(-1)^{q+K}(q-K)\left(\begin{array}{l}
h \\
K
\end{array}\right)=(-1)^{q} \sum_{0 \leq K \leq h}(-1)^{K}(q-K)\left(\begin{array}{l}
h \\
K
\end{array}\right)=0
$$

by $h \geq 2$. Thus we have completed the proof.

\section{References}

Duke, W., Friedlander, J. B., \& Iwaniec, H. (1995). Equidistribution of roots of a quadratic congruence to prime moduli. Ann. of Math., 141, 423-441. https://doi.org/10.2307/2118527

Feller, W. (1966). An introduction to probability theory and its applications. vol. 2, New York, J. Wiley.

Kitaoka, Y. (2017). Notes on the distribution of roots modulo a prime of a polynomial. Unif. Distrib. Theory, 12, 91-117.

Tóth. Á. (2000). Roots of Quadratic congruences, Internat. Math. Res. Notices, 719-739. https://doi.org/10.1155/S1073792800000404

\section{Copyrights}

Copyright for this article is retained by the author(s), with first publication rights granted to the journal.

This is an open-access article distributed under the terms and conditions of the Creative Commons Attribution license (http://creativecommons.org/licenses/by/4.0/). 